\title{
Classification of maximally supersymmetric backgrounds in supergravity theories
}

\section{Jan Louis and Severin Lüst}

Fachbereich Physik der Universität Hamburg, Luruper Chaussee 149, 22761 Hamburg, Germany

Zentrum für Mathematische Physik, Universität Hamburg, Bundesstrasse 55, D-20146 Hamburg, Germany

E-mail: jan.louis@desy.de, severin.luest@desy.de

ABSTRACT: We study maximally supersymmetric solutions of all gauged or deformed supergravity theories in $D \geq 3$ space-time dimensions. For vanishing background fluxes the space-time background has to be either Minkowski or anti-de Sitter. We derive a simple criterion for the existence of solutions with non-trivial fluxes and determine all supergravities that satisfy it. We show that their solutions coincide with those of the corresponding ungauged theories and conclude that the known list of maximally supersymmetric solutions is exhaustive.

KEYwords: Extended Supersymmetry, Supergravity Models, Superstring Vacua

ARXIV EPRINT: 1607.08249 


\section{Contents}

1 Introduction 1

2 Preliminaries 2

$\begin{array}{lll}3 & \text { Supersymmetric backgrounds without fluxes } & 6\end{array}$

$\begin{array}{lll}4 & \text { Supersymmetric backgrounds with fluxes } & 7\end{array}$

5 Conclusions 12

$\begin{array}{ll}\text { A } \Gamma \text {-matrix conventions } & 13\end{array}$

$\begin{array}{ll}\text { B Analysis of the integrability condition } & 14\end{array}$

$\begin{array}{ll}\text { C The gauged R-symmetry connection } & 15\end{array}$

\section{Introduction}

Classical solutions of supergravity theories have been intensely studied. Of particular interest are supersymmetric bosonic solutions where the supersymmetry variations of all fermionic fields present in the theory vanish. These have been classified in many cases, nevertheless a complete description of all supersymmetric solutions has not been obtained yet. ${ }^{1}$ In this paper we exclusively focus on maximally supersymmetric solutions, that is solutions which preserve all supercharges of a given supergravity. In this case the resulting Killing spinor equations have to admit an independent Killing spinor for each supercharge which considerably constrains the allowed space-time backgrounds.

If one excludes background fluxes, the Killing spinor equations take a particularly simple form and can be integrated directly. In this case one finds that in supergravities with $D$ space-time dimensions only two backgrounds are possible: $D$-dimensional Minkoswki space $M_{D}$ or $D$-dimensional anti-de Sitter space $\operatorname{AdS}_{D}$. While $M_{D}$ is a solution of all ungauged supergravities, $\mathrm{AdS}_{D}$ backgrounds require a non-trivial scalar potential and hence the supergravities have to be gauged or otherwise deformed. ${ }^{2}$ It turns out that generically additional algebraic conditions have to be satisfied which further restrict the gauged and/or deformed supergravity.

More complicated solutions only arise if non-trivial background fluxes of gauge potentials in the gravitational multiplet are turned on. However, these fluxes generically enter the supersymmetry variation of the spin- $1 / 2$ fermions in the gravitational multiplet and break supersymmetry (at least partially). The only exception occurs for gauge potentials

\footnotetext{
${ }^{1}$ For the ungauged case see for example $[1-26]$ and [27-39] for the gauged case.

${ }^{2}$ By deformed supergravities we denote theories which are deformed by mass parameters or the superpotential in $D=4, \mathcal{N}=1$ supergravity, for example.
} 
with (anti-)self-dual field strengths in a chiral theory which drop out of the spin- $1 / 2$ variations. As we will see, this limits the possible supergravities to a small subset where either the gravitational multiplet does not contain spin- $1 / 2$ fermions or the theory is chiral and features (anti-)self-dual fields.

We further show that in the presence of non-trivial fluxes the background values of the supersymmetry variations have to coincide with the supersymmetry variations of the corresponding ungauged theories. Concretely this means that the fermionic shift matrices as well as the gauge connection for the R-symmetry have to vanish in the background. ${ }^{3}$ We prove this property by deriving a generic expression for the R-symmetry connection. As a by-product we show that in maximally supersymmetric backgrounds the R-symmetry can only be gauged by vector fields of the gravitational multiplet. ${ }^{4}$

The correspondence with the ungauged theories implies that also the background solutions coincide. It turns out that for all situation where background fluxes are possible the maximally supersymmetric solutions have already been determined and classified $[1,4,5,8,9]$. They are either space-times of the Freund-Rubin form $A d S_{d} \times S^{(D-d)}$ [40] or $\mathrm{H} p p$-wave solutions $[41,42]$. Only in five space-time dimensions one can have more exotic solutions [4]. Although all these solution were known previously our analysis shows that this list is exhaustive.

This paper is organized as follows. In section 2 we set the stage for our analysis and recall the supersymmetry transformations of the fermionic fields. In particular we establish a notation which allows us to discuss the various supergravities in a common framework. In section 3 we show that supergravities with $D$-dimensional space-times without fluxes have supersymmetric backgrounds that are either $\mathrm{AdS}_{D}$ or $M_{D}$. In section 4 we turn on background fluxes and argue that the vanishing of the supersymmetry transformation of the spin- $\frac{1}{2}$ fermions requires that all background fluxes are zero except in chiral theories with (anti-)self-dual fluxes. We further show that solutions with non-trivial fluxes coincide with the solutions of the corresponding ungauged theories. Some of the technical analysis is relegated to three appendices. In appendix A we summarize our $\Gamma$-matrix conventions, in appendix B we supply some of the technical details necessary in section 4 and finally in appendix $\mathrm{C}$ we determine the general gauging of the R-symmetry in maximally supersymmetric backgrounds.

\section{Preliminaries}

In this paper we discuss properties of supergravity backgrounds in arbitrary space-time dimensions and for varying number of supercharges in a given dimension. In order to avoid a case-by-case analysis we introduce a unifying notation which allows us to more or less discuss all cases simultaneously. It is the purpose of this section to set the stage for this analysis and provide a common notation.

\footnotetext{
${ }^{3}$ This result has been obtained previously for gauged $D=4, \mathcal{N}=2$ supergravity in [28, 37], for minimal gauged $D=5, \mathcal{N}=2$ supergravity in [27] and for $D=6, \mathcal{N}=(1,0)$ supergravity in [26]. However theses results always rely on the specific formulation of the particular gauged supergravities under consideration.

${ }^{4}$ In the generic situation it is precisely speaking not the R-symmetry which is being gauged but a subgroup of the scalar field space's isometry group which in turn induces R-symmetry transformations. For a more detailed discussion see the explanations around (2.14) and also in appendix C.
} 
Supergravities in $D$ space-time dimensions contain a gravitational multiplet whose generic field content includes the metric $g_{M N}, M, N=0, \ldots, D-1, \mathcal{N}$ gravitini $\psi_{M}^{i}$, $i=1, \ldots, \mathcal{N}$, a set of $(p-1)$-form gauge potentials $A^{(p-1)}$ (with $p$-form field strengths $F^{(p)}$ ), a set of spin- $\frac{1}{2}$ fermions $\chi^{a}$ as well as a set of scalar fields $\phi$. Note that not all of these component fields necessarily have to be part of a given gravitational multiplet but we gave the most general situation. Moreover, there might be additional multiplets in the spectrum (e.g. vector, tensor or matter multiplets) which can also have gauge potentials among their component members. For the moment we denote all gauge potentials by $A^{(p-1)}$ but we distinguish them shortly. The spin- $\frac{1}{2}$ fermions in the extra multiplets we collectively call $\lambda^{s}$ while all scalars we universally denote as $\phi$.

Let us first focus on the kinetic terms for the gauge potentials. They are of the generic form (for a review see, for example, [43])

$$
\mathcal{L}_{\text {kin }}=-\frac{1}{2} \sum_{p} M_{I_{p} J_{p}}^{(p)}(\phi) F^{(p) I_{p}} \wedge * F^{(p) J_{p}},
$$

where the indices $I_{p}, J_{p}$ label field strengths of the same rank $p$ and the sum runs over all possible $p$-forms which are present in a given theory. The matrices $M^{(p)}$ generically depend on all scalar fields, are symmetric and positive definite. Therefore they can be diagonalized via

$$
M_{I_{p} J_{p}}^{(p)}=\delta_{\alpha_{p} \beta_{p}} \mathcal{V}_{I_{p}}^{\alpha_{p}} \mathcal{V}_{J_{p}}^{\beta_{p}}
$$

where the vielbeins $\mathcal{V}_{I_{p}}^{\alpha_{p}}$ are again scalar dependent. Later on it will be important to distinguish which of the form fields enter the supersymmetry variations of the gravitini. For this purpose we introduce the abbreviation ${ }^{5}$

$$
F^{\alpha_{p}}=\mathcal{V}_{I_{p}}^{\alpha_{p}} F^{I_{p}}
$$

and split the indices $\alpha_{p}$ according to

$$
\alpha_{p}=\left(\hat{\alpha}_{p}, \tilde{\alpha}_{p}\right)
$$

We then denote by $F^{\hat{\alpha}_{p}}$ the field strengths in the gravitational multiplet (e.g. the graviphotons for $p=2$ ) and by $F^{\tilde{\alpha}_{p}}$ the field strengths of gauge potentials which arise in all other multiplets that might be present. Note that this split depends on the scalar fields via the vielbeins $\mathcal{V}$ and thus is background dependent.

After this preparation we recall the supersymmetry variations of the fermions, which are of special importance in the following. The transformation of the gravitini takes the generic form

$$
\delta \psi_{M}^{i}=\mathrm{D}_{M} \epsilon^{i}+\left(\mathcal{F}_{M}\right)_{j}^{i} \epsilon^{j}+A_{0 j}^{i} \Gamma_{M} \epsilon^{j}
$$

where

$$
\mathrm{D}_{M} \epsilon^{i}=\nabla_{M} \epsilon^{i}-\left(\mathcal{Q}_{M}\right)_{j}^{i} \epsilon^{j}
$$

\footnotetext{
${ }^{5}$ In the following we frequently drop the labels $(p)$ in order to not overload the notation.
} 
$\nabla_{M}$ denotes the Levi-Cevita connection and $\mathcal{Q}_{M}$ is the R-symmetry connection which we discuss in more detail shortly. The second term in (2.5) contains the various field strengths and is given by

$$
\left(\mathcal{F}_{M}\right)_{j}^{i}=\sum_{p \geq 2} \sum_{\hat{\alpha}_{p}}\left(B_{\hat{\alpha}_{p}}^{(p)}\right)_{j}^{i} F_{N_{1} \ldots N_{p}}^{\hat{\alpha}_{p}} T_{(p)}^{N_{1} \ldots N_{p}}{ }_{M}
$$

where the $B^{(p)}$ are constant matrices. The matrices $T_{(p)}^{N_{1} \ldots N_{p}} M$ are defined as

$$
T_{(p)}^{N_{1} \ldots N_{p}}{ }_{M}=\Gamma^{N_{1} \ldots N_{p}}{ }_{M}+\beta_{(p)} \Gamma^{\left[N_{1} \ldots N_{p-1}\right.} \delta_{M}^{\left.N_{p}\right]},
$$

where $\Gamma^{N_{1} \ldots N_{p}}$ is an antisymmetrized product of $\Gamma$-matrices (see appendix A for our conventions) and

$$
\beta_{(p)}=\frac{p(D-p-1)}{p-1} .
$$

Finally, the matrix $A_{0}$ in the third term of (2.5) arises in gauged and/or deformed supergravities and is parameterized by the gaugings or deformations and in general depends on all scalar fields in the spectrum [43]. Its precise form is specific to the supergravity under consideration.

Let us now turn to the spin- $\frac{1}{2}$ fermions $\chi^{a}$ and $\lambda^{s}$. The $\chi^{a}$ are part of the gravitational multiplet and their transformations take the generic form

$$
\delta \chi^{a}=\sum_{p \geq 1} \sum_{\hat{\alpha}_{p}}\left(C_{\hat{\alpha}_{p}}^{(p)}\right)_{i}^{a} F_{N_{1} \ldots N_{p}}^{\hat{\alpha}_{p}} \Gamma^{N_{1} \ldots N_{p}} \epsilon^{i}+A_{1 i}^{a} \epsilon^{i} .
$$

The $\lambda^{s}$ are members of other multiplets present (e.g. vector-, tensor- or matter-multiplets) and we similarly have

$$
\delta \lambda^{s}=\sum_{p \geq 1} \sum_{\tilde{\alpha}_{p}}\left(D_{\tilde{\alpha}_{p}}^{(p)}\right)_{i}^{s} F_{N_{1} \ldots N_{p}}^{\tilde{\alpha}_{p}} \Gamma^{N_{1} \ldots N_{p}} \epsilon^{i}+A_{2 i}^{s} \epsilon^{i} .
$$

Note that the field strengths appearing in (2.10) and (2.11) form a disjoint set. Accordingly they are labeled by $\hat{\alpha}_{p}, \tilde{\alpha}_{p}$ that we introduced in (2.4). Contrary to (2.7) the sums in (2.10) and (2.11) start already at $p=1$ and thus include the fields strengths of the scalar fields $F_{M}^{\alpha_{1}}=D_{M} \phi^{\alpha_{1}}$ which do not enter the gravitino variations (2.5). As in the gravitino variations $C^{p}$ and $D^{p}$ are constant matrices while $A_{1}$ and $A_{2}$ arise in gauged supergravities and depend on the gaugings/deformations and the scalar fields. They have a specific form in a given supergravity. Supersymmetry relates the fermionic shift matrices $A_{0}, A_{1}$ and $A_{2}$ to the scalar potential $V$ and generically one has

$$
V=-c_{0} \operatorname{tr}\left(A_{0}^{\dagger} A_{0}\right)+c_{1} \operatorname{tr}\left(A_{1}^{\dagger} A_{1}\right)+c_{2} \operatorname{tr}\left(A_{2}^{\dagger} A_{2}\right),
$$

where $c_{0}, c_{1}$ and $c_{2}$ are numerical constants fixed by supersymmetry in a given supergravity.

Let us return to the connection $\mathcal{Q}_{M}$ in the covariant derivative (2.6) as it will play an important role in the following and we need to establish some of its properties. In a generic supergravity $\mathcal{Q}_{M}$ splits according to [43-45]

$$
\mathcal{Q}_{M}=\mathcal{Q}_{M}^{\text {scalar }}+\mathcal{Q}_{M}^{\text {gauge }},
$$


where $\mathcal{Q}_{M}^{\text {scalar }}$ is a composite connection which only depends on the scalar fields and their derivatives and already exists in the ungauged theory. A transformation along a possible isometry of the scalar field space can induce a scalar field dependent R-symmetry transformation and the connection term $Q_{M}^{\text {scalar }}$ is necessary to make $\mathrm{D}_{M} \epsilon^{i}$ transform covariantly. ${ }^{6}$ These transformations can be made local (i.e. not only scalar field but also explicitly spacetime dependent) by introducing another term $\mathcal{Q}_{M}^{\text {gauge }}$ which contains a linear combination of gauge fields $A^{\alpha_{2}}$, i.e.

$$
\mathcal{Q}_{M}^{\text {gauge }}=A_{M}^{\alpha_{2}} t_{\alpha_{2}} .
$$

The matrices $t_{\alpha_{2}}$ are often called moment maps and generically depend again on the scalar fields. They take values in the Lie-algebra $\mathfrak{g}_{R}$ of the R-symmetry group, but at most points in field space they do not need to span a proper Lie-subalgebra of $\mathfrak{g}_{R}$. However, we will show in appendix $\mathrm{C}$ that the $t_{\alpha_{2}}$ close under the action of the Lie-bracket in every maximally supersymmetric background. In this specific situation we say for simplicity that the subgroup of the R-symmetry group which is generated by the background values of the $t_{\alpha_{2}}$ is gauged in this background, or shortly that the R-symmetry is gauged (in this background). As explained above it would be however generically more precise to speak about gauging a certain subgroup of the scalar manifold's isometry group which in turn induces R-symmetry transformations. Moreover, let us stress that in principle all gauge fields, those from the gravitational multiplet (the graviphotons) as well as gauge fields from other multiplets (e.g. vector multiplets), can appear in (2.14).

In the following we also need the curvature or field strength $\mathcal{H}_{M N}$ of $\mathcal{Q}_{M}$. As usual it appears in the commutator of the covariant derivatives defined in (2.6) as follows

$$
\left[\mathrm{D}_{M}, \mathrm{D}_{N}\right] \epsilon^{i}=\frac{1}{4} R_{M N P Q} \Gamma^{P Q} \epsilon^{i}-\left(\mathcal{H}_{M N}\right)_{j}^{i} \epsilon^{j},
$$

where $R_{M N P Q}$ is the Riemann tensor of the background space-time $\mathcal{M}_{D}$. As a consequence of (2.13) $\mathcal{H}_{M N}$ similarly decomposes as

$$
\mathcal{H}_{M N}=\mathcal{H}_{M N}^{\text {scalar }}+\mathcal{H}_{M N}^{\text {gauge }}
$$

with

$$
\mathcal{H}_{M N}^{\text {gauge }}=F_{M N}^{\alpha_{2}} t_{\alpha_{2}},
$$

and $t_{\alpha_{2}}$ being the same matrices as in (2.14). The field strength of the composite connection $\mathcal{H}_{M N}^{\text {scalar }}$ can be expressed in terms of the field strengths of the scalar fields $F_{M}^{\alpha_{1}}$ and takes the generic form

$$
\mathcal{H}_{M N}^{\text {scalar }}=h_{1} C_{\hat{\alpha}_{1}}^{\dagger} C_{\hat{\beta}_{1}} F_{[M}^{\hat{\alpha}_{1}} F_{N]}^{\hat{\beta}_{1}}+h_{2} D_{\tilde{\alpha}_{1}}^{\dagger} D_{\tilde{\beta}_{1}} F_{[M}^{\tilde{\alpha}_{1}} F_{N]}^{\tilde{\beta}_{1}},
$$

where $C, D$ are the matrices appearing in (2.10) for $p=1$ and (2.11) respectively and $h_{1}$ and $h_{2}$ are numerical constants determined by supersymmetry in a given supergravity.

\footnotetext{
${ }^{6}$ To be more specific let us assume that the scalar fields $\phi$ span some manifold $\mathcal{T}$, i.e. $\phi: \mathcal{M}_{D} \rightarrow \mathcal{T}$, where $\mathcal{M}_{D}$ denotes the space-time manifold. Generically the gravitini and hence also the supersymmetry parameters $\epsilon^{i}$ are sections of a (non-trivial) vector bundle over $\mathcal{T}$ with connection $\omega$. Then $\mathcal{Q}^{\text {scalar }}$ is just the pullback of this connection with respect to $\phi$, i.e. $\mathcal{Q}^{\text {scalar }}=\phi^{*} \omega$.
} 
Let us close this section by recalling that a supergravity background which preserves some supersymmetry has to admit spinors $\epsilon^{i}$ which satisfy

$$
\delta \psi_{M}^{i}=\delta \chi^{a}=\delta \lambda^{s}=0 .
$$

The number of linearly independent such spinors then determines the number of preserved supercharges. In this paper we only consider backgrounds which preserve all supercharges of the supergravity under consideration. This considerably simplifies the analysis as we will see shortly.

\section{Supersymmetric backgrounds without fluxes}

Let us first analyze the situation where all background fluxes vanish and hence eqs. (2.5)-(2.11) simplify. If all supercharges are preserved, $\delta \chi^{a}=\delta \lambda^{s}=0$ imply via (2.10) and (2.11) that ${ }^{7}$

$$
A_{1}=A_{2}=0 .
$$

On the other hand, the vanishing of the gravitino variation (2.5)

$$
\delta \psi_{M}^{i}=\mathrm{D}_{M} \epsilon^{i}+A_{0 j}^{i} \Gamma_{M} \epsilon^{j}=0
$$

says that $\epsilon^{i}$ has to be a Killing spinor. Its existence implies a strong constraint on the space-time manifold which can be derived by acting with another covariant derivative, antisymmetrizing and using (2.15). This implies

$$
\left[\left(\frac{1}{4} R_{M N}{ }^{P Q} \delta_{k}^{i}+2 A_{0 j}^{i} A_{0 k}^{j} \delta_{M}^{P} \delta_{N}^{Q}\right) \Gamma_{P Q}+2\left(\mathrm{D}_{[M} A_{0}\right)_{k}^{i} \Gamma_{N]}\right] \epsilon^{k}=0,
$$

where we also used that $\mathcal{H}_{M N}$ vanishes in backgrounds without any fluxes and where the covariant derivative of $A_{0}$ is defined as $\mathrm{D}_{M} A_{0}=\partial_{M} A_{0}-\left[\mathcal{Q}_{M}, A_{0}\right]$. In a background which preserves all supercharges the expression in the bracket has to vanish at each order in the $\Gamma$ matrices independently. From the term linear in $\Gamma$ we learn that $A_{0}$ is covariantly constant. The part quadratic in $\Gamma$ then says that $A_{0}^{2}$ needs to be proportional to the identity matrix and must be a constant since

$$
\partial_{M} A_{0}^{2}=\mathrm{D}_{M} A_{0}^{2}=0
$$

Moreover it implies that in a given supergravity the maximally supersymmetric backgrounds have to be maximally symmetric space-times with a Riemann tensor given by

$$
R_{M N P Q}=-\frac{4}{\mathcal{N}} \operatorname{tr}\left(A_{0}^{2}\right)\left(g_{M P} g_{N Q}-g_{M Q} g_{N P}\right) .
$$

From the canonical Einstein equations one readily infers that in such backgrounds the cosmological constant $\Lambda$ is given by

$$
\Lambda=-\frac{2}{\mathcal{N}}(D-1)(D-2) \operatorname{tr}\left(A_{0}^{2}\right),
$$

\footnotetext{
${ }^{7}$ Both equations only have to hold in the background, i.e. the conditions read $\left\langle A_{1}\right\rangle=\left\langle A_{2}\right\rangle=0$. However, in order to keep the notation manageable we generically omit the brackets henceforth.
} 
and the background value of the scalar potential is related by $\langle V\rangle=\Lambda$. Note that consistency then determines the coefficient $c_{0}$ of $V$ in $(2.12)$ to be $c_{0}=\frac{2}{\mathcal{N}}(D-1)(D-2)$. For $A_{0} \neq 0$ we thus have an AdS-background $\mathcal{M}_{D}=\mathrm{AdS}_{D}$ while for $A_{0}=0$ the background is flat. So altogether fully supersymmetric backgrounds without background fluxes have to be one of the following cases

$$
\mathcal{M}_{D}=\operatorname{AdS}_{D} \quad \text { or } \quad \mathcal{M}_{D}=M_{d} \times T^{(D-d)}, \quad 1 \leq d \leq D,
$$

up to local isometries. We see in particular that without fluxes supersymmetric backgrounds with an $\mathrm{AdS}_{d}$ factor cannot exist for $d<D$.

Before we proceed let us note that in a given $D$-dimensional gauged supergravity the existence of the $\mathcal{M}_{D}=\mathrm{AdS}_{D}$ background requires the existence of a solution with

$$
A_{0}^{2}=-\frac{\Lambda}{2(D-1)(D-2)} \mathbb{1}, \quad A_{1}=A_{2}=0 .
$$

This can only be checked in a case-by-case analysis and explicit solutions have indeed been constructed in a variety of supergravities (see, for example, [37, 46-50] and references therein). However, from ref. [51] it is known that AdS superalgebras only exist for $D<8$ and in $D=6$ only for the non-chiral $\mathcal{N}=(1,1)$ supergravity. In the other cases no solution of (3.8) can exist.

\section{Supersymmetric backgrounds with fluxes}

In this section we extend our previous analysis in that we consider backgrounds with non-trivial fluxes and reanalyze the implications for the possible space-time manifolds. In this case the vanishing supersymmetry variations of the spin-1/2 fermions given in (2.10) and (2.11) immediately impose additional constraints. As we will see, they are particularly strong for the fermions $\chi^{a}$ in the gravitational multiplet. Since the $\Gamma$-matrices and their antisymmetric products are linearly independent, $\delta \chi^{a}=\delta \lambda^{s}=0$ enforces

$$
A_{1}=A_{2}=0 \quad \text { and } \quad F^{(p)}=0,
$$

for all possible values of $p .{ }^{8}$ This seems to imply that no background fluxes can be turned on. However, this conclusion can be evaded either if there simply are no spin- $1 / 2$ fermions in the gravity multiplet or if there is an (anti-)self-dual field strength in a chiral theory.

In the first case there is no condition on the fluxes $F^{\hat{\alpha}_{p}}$ which appear in the gravitino variation (2.5) and (2.7) but only on the fluxes $F^{\tilde{\alpha}_{p}}$ which feature in (2.11). The second exception follows from the definition of the chirality operator $\Gamma_{*}$ (given in (A.3)) which implies that in even dimensions $D$ the Hodge-dual of a $p$-form $F^{(p)}$ satisfies

$$
* F^{(p)} \cdot \Gamma=-(-1)^{p(p-1) / 2} i^{D / 2+1}\left(F^{(p)} \cdot \Gamma\right) \Gamma_{*},
$$

\footnotetext{
${ }^{8}$ In even dimensions $D$ all antisymmetric products of gamma matrices are linearly independent while in odd dimensions only those up to rank $(D-1) / 2$ are linearly independent as can bee seen from (A.5). This however is strong enough to enforce (4.1).
} 


\begin{tabular}{|c|c|c|c|c|}
\hline dimension & supersymmetry & $\mathrm{q}$ & possible flux & ref. \\
\hline$D=11$ & $\mathcal{N}=1$ & 32 & $F^{(4)}$ & {$[5]$} \\
$D=10$ & IIB & 32 & $F_{+}^{(5)}$ & {$[5]$} \\
$D=6$ & $\mathcal{N}=(2,0)$ & 16 & $5 \times F_{+}^{(3)}$ & {$[9]$} \\
$D=6$ & $\mathcal{N}=(1,0)$ & 8 & $F_{+}^{(3)}$ & {$[8]$} \\
$D=5$ & $\mathcal{N}=2$ & 8 & $F^{(2)}$ & {$[4]$} \\
$D=4$ & $\mathcal{N}=2$ & 8 & $F^{(2)}$ & {$[1]$} \\
\hline
\end{tabular}

Table 1. Supergravity theories which allow for a background flux that does not break supersymmetry. $q$ denotes the number of real supercharges. In the last column we give the reference for the classification of maximally supersymmetric solutions.

where we abbreviated $F^{(p)} \cdot \Gamma=F_{N_{1} \ldots N_{p}}^{(p)} \Gamma^{N_{1} \ldots N_{p}}$ (and used (A.4)). Note that the prefactor is real in dimensions $D=2 \bmod 4$, which are precisely those dimensions in which chiral theories can exist. In these dimensions one finds for an (anti-)self-dual $D / 2$-form $F_{ \pm}=$ $\pm * F_{ \pm}$that

$$
F_{ \pm} \cdot \Gamma=\left(F_{ \pm} \cdot \Gamma\right) P_{ \pm}
$$

where $P_{ \pm}=\frac{1}{2}\left(\mathbb{1} \pm \Gamma_{*}\right)$. In the chiral supergravities in $D=6,10$ [52-55] the supergravity multiplet contains two or four-form fields, respectively, with self-dual field strengths $F_{+}^{\hat{\alpha}_{D / 2}}$. In these theories the gravitini and consequently also the supersymmetry parameters $\epsilon^{i}$ are left-handed. Therefore, a term of the form $\left(F_{+}^{\hat{\alpha}_{D / 2}} \cdot \Gamma\right) \epsilon^{i-}$ cannot appear in (2.10) which indeed shows that a non-vanishing background value for a self-dual field strength does not break supersymmetry in these theories. Nevertheless $F_{+}^{\hat{\alpha}_{D / 2}}$ still enters the variation of the gravitini, as a different contraction with $\Gamma$-matrices appears in (2.7). Hence maximally supersymmetric solutions with non-trivial background flux are possible.

The previous considerations in this section enable us to conclude that solutions which preserve all supercharges of a given supergravity and which are different from the ones described in the previous section can only exist if at least one of the following two conditions hold:

Either the gravity multiplet contains p-form gauge fields but no spin- $\frac{1}{2}$ fermions $\chi^{a}$ or the theory is chiral and (some of) the gauge potentials in the gravity multiplet satisfy an (anti-) self-duality condition such that they drop out of $\delta \chi^{a}$.

In table 1 we list all possible supergravities in dimensions $D \geq 3$ which satisfy these conditions, together with the possible background fluxes. ${ }^{9}$ We now proceed by analyzing the supersymmetry variation of the gravitini (2.5) for these theories in more detail.

\footnotetext{
${ }^{9}$ It is in fact easy to see that such theories cannot exist in $D=3$ dimensions. Since three-dimensional gravity is non-dynamical, the graviton, and via supersymmetry also the gravitini, do not carry any on-shell degrees of freedom. So whenever the gravity multiplet contains vector or scalar fields (which are dual in three dimensions) it must also contain spin- $1 / 2$ fields as supersymmetric partners.
} 
Taking a covariant derivative of (2.5) and using (2.15) we arrive at the integrability condition

$$
\begin{aligned}
\left(\frac{1}{4} R_{M N P Q} \Gamma^{P Q} \delta_{j}^{i}-\left(\mathcal{H}_{M N}\right)_{j}^{i}+2\left(\mathrm{D}_{[M} \mathcal{F}_{N]}+\mathrm{D}_{[M} A_{0} \Gamma_{N]}\right)_{j}^{i}\right. \\
\left.\quad+\left[\left(\mathcal{F}_{M}+A_{0} \Gamma_{M}\right)_{k}^{i}\left(\mathcal{F}_{N}+A_{0} \Gamma_{N}\right)_{j}^{k}-(M \leftrightarrow N)\right]\right) \epsilon^{j}=0 .
\end{aligned}
$$

In a maximally supersymmetric background this has to vanish at each order in the $\Gamma$ matrices independently. As we show in appendix B for all the theories in table 1 the only term at zeroth order in $\Gamma$ is $\mathcal{H}_{M N}$ and thus we arrive at

$$
\mathcal{H}_{M N}=0 \text {. }
$$

Furthermore, due to (4.1) all scalar fields have vanishing field strengths, $F^{\hat{\alpha}_{1}}=F^{\tilde{\alpha}_{1}}=0$, and therefore, using (2.18), $\mathcal{H}_{M N}^{\text {scalar }}$ automatically vanishes. From (2.16) we then learn that (4.5) implies

$$
\mathcal{H}_{M N}^{\text {gauge }}=0 \text {. }
$$

In a next step we show that (4.6) says that there can be either no background fluxes at all or that alternatively both $A_{0}$ and $\mathcal{Q}_{M}^{\text {gauge }}$ vanish in the background. To see this we derive in appendix $\mathrm{C}$ that the supersymmetry conditions $A_{1}=A_{2}=0$ of (4.1) enforce $\mathcal{H}_{M N}^{\text {gauge }}$ to be of the generic form

$$
\mathcal{H}_{M N}^{\text {gauge }} \sim F_{M N}^{\hat{\alpha}_{2}}\left\{A_{0}, B_{\hat{\alpha}_{2}}\right\}
$$

where the precise factor of proportionality is given in (C.8) but is not important for the following discussion. Due to (2.14) and (2.17) the same relation holds for $Q_{M}^{\text {gauged }}$ with $F_{M N}^{\hat{\alpha}_{2}}$ replaced by $A_{M}^{\hat{\alpha}_{2}}$.

Eq. (4.7) has a few notable features. First of all the appearance of $F_{M N}^{\hat{\alpha}_{2}}$ says that in the background the R-symmetry can only be gauged (in the sense discussed below (2.14)) by graviphotons, i.e. by vector fields in the gravity multiplet. ${ }^{10}$ Moreover, (4.7) uniquely determines the gauged subgroup of the R-symmetry group for maximally supersymmetric vacua and gives an explicit formula for its computation in terms of $A_{0}$ and $B_{\hat{\alpha}_{2}}$. We finally want to stress that this is a generic result, not restricted to the theories in table 1 but true for all gauged supergravity theories with $D \geq 4$.

Let us study the implications of (4.7) for the supergravities of table 1. We already showed that the theories which are not in this list cannot have non-vanishing background fluxes so that (4.6) is trivially satisfied and does not impose any conditions on $A_{0}$. Similarly, for the first three theories in table 1 it is known that deformations by a non-vanishing $A_{0}$ do not exist. In addition no massless vector fields appear in the gravitational or in any other multiplet. Hence $H_{M N}^{\text {gauge }}$ and $Q_{M}^{\text {gauge }}$ do not exist and the theories are always ungauged,

\footnotetext{
${ }^{10}$ This is intuitively plausible but we are not aware of any previous general proof. Moreover notice that this is a priori only a statement about the maximally supersymmetric background. At an arbitrary point in field space other gaugings might be in principle possible. See also the discussion at the beginning of appendix C.
} 
consistent with (4.7). On the other hand the possible background fluxes of higher rank field strengths are not restricted. Similarly, the six-dimensional $\mathcal{N}=(1,0)$ theories cannot be deformed by $A_{0} \neq 0$ and do not feature any vector fields in the gravity multiplet. In principle it is possible to gauge these theories by coupling them to vector multiplets. However, in the maximally supersymmetric background this is forbidden due to (4.7) and therefore also here $Q_{M}^{\text {gauge }}=0$ holds. This was explicitly shown in [26].

The analysis of the two remaining supergravities in the list, the four- and fivedimensional $\mathcal{N}=2$ theories, is slightly more involved. Both can be deformed by $A_{0} \neq 0$ and both have one single gauge field, the graviphoton $A^{\hat{\alpha}_{2}}$, in the gravity multiplet. Consequently there is also only one single matrix $B_{\hat{\alpha}_{2}}$. As the graviphoton is an R-symmetry singlet, $B_{\hat{\alpha}_{2}}$ has to be proportional to the identity. Therefore (4.7) simply reads

$$
\mathcal{H}_{M N}^{\text {gauge }} \sim F_{M N} A_{0},
$$

where $F_{M N}$ is the field strength of the graviphoton. As a consequence, (4.6) implies that either $F_{M N}$ or $A_{0}$ has to vanish in the background. For $N=2$ theories in $D=4$ this has been explicitly shown for pure gauged supergravity in [28] and for arbitrary gauging in [37]. For pure gauged supergravity in $D=5$ this has been obtained in [27] and related results for arbitrary gaugings in [35]. In contrast to their results our analysis here is completely general and does not rely on the concrete formulation of the gauged supergravities.

Let us summarize our results so far. There are two different branches of maximally supersymmetric solutions:

i) $A_{0} \neq 0$.

In this case all background fluxes must necessarily vanish and the background spacetime is $A d S_{D}$ as described in section 3 .

ii) $A_{0}=0$

In this case non-vanishing background fluxes are allowed but $Q_{M}$ vanishes in the background. As a consequence the fermionic supersymmetry transformation (2.5) take exactly the same form as for the ungauged theory and hence the maximally supersymmetric solutions coincide with the solutions of the ungauged theories.

The solutions of the ungauged theories have been classified for all supergravities listed in table 1 and this classification can thus be used for case ii). These solutions can be found in the references given in table 1 . Let us shortly review the main results. For vanishing $A_{0}$ and $Q_{M}$ the integrability condition (4.4) simplifies considerably and reads

$$
\frac{1}{4} R_{M N P Q} \Gamma^{P Q} \delta_{j}^{i}+2\left(\nabla_{[M} \mathcal{F}_{N]}\right)_{j}^{i}+2\left(\mathcal{F}_{[M}\right)_{k}^{i}\left(\mathcal{F}_{N]}\right)_{j}^{k}=0
$$

Expanding in powers of the $\Gamma$-matrices and collecting all terms quadratic in $\Gamma$ we observe that the Riemann tensor of the space-time background is expressed solely in terms of the background flux $F^{\hat{\alpha}_{p}}$ and its derivatives. Furthermore, all supergravities listed in table 1 have solutions with the property

$$
\nabla F^{\hat{\alpha}_{p}}=0 .
$$


Only in the five-dimensional $\mathcal{N}=2$ supergravity one finds solutions of (4.9) which do not satisfy (4.10) [4]. In all other cases there are no additional solutions or in other words all solutions share the property (4.10). For these solutions also the Riemann tensor is parallel, i.e. $\nabla_{M} R_{N P Q R}=0$, which says that the space-time is locally symmetric. The locally symmetric spaces with Lorentzian signature are classified $[5,56] .{ }^{11}$ Furthermore, in $[5,8,9]$ it was shown that $F^{\hat{\alpha}_{p}}$ can be written as

$$
F^{\hat{\alpha}_{p}}=v^{\hat{\alpha}_{p}} F \quad \text { or } \quad F^{\hat{\alpha}_{p}}=v^{\hat{\alpha}_{p}}(F+* F),
$$

where $v^{\hat{\alpha}_{p}}$ is constant and $F$ is decomposable, i.e. it can always be expressed as the wedgeproduct of $p$ one-forms. The second decomposition holds for a self-dual $F^{\hat{\alpha}_{p}} \cdot{ }^{12}$ Excluding the trivial case where $F=0$ and where the background is flat, there are therefore only two cases to be distinguished:

1. $F$ is not a null form (i.e. $F^{2} \neq 0$ ).

These are the well-known solutions of Freund-Rubin type [40] for which the spacetime is the product of an AdS space and a sphere such that $F$ is a top-form on one of the two factors, i.e.

$$
\mathcal{M}_{D}=\operatorname{AdS}_{p} \times S^{(D-p)} \quad \text { or } \quad \mathcal{M}_{D}=\operatorname{AdS}_{(D-p)} \times S^{p} .
$$

We explicitly list all these solutions in table 2. Notice that besides the pure $\operatorname{AdS}_{D}$ solutions discussed in section 3 these are the only possible maximally supersymmetric solutions with an AdS-factor. All other AdS solutions in supergravity will necessarily break supersymmetry.

2. $F$ is a null form (i.e. $F^{2}=0$ ).

These solutions are homogeneous $p p$-waves (H $p p$-waves) first discovered by KowalskiGlikman [41, 42] and therefore often referred to as KG solutions. They can be obtained from the respective AdS $\times S$ solutions by a Penrose limit [57-60].

As we have already mentioned above this list of solutions is exhaustive if one excludes the five-dimensional $\mathcal{N}=2$ supergravity. In the latter theory there can be more exotic solutions with $F$ not parallel or decomposable and consequently also the background spacetime $\mathcal{M}_{D}$ not locally symmetric. These exceptional solutions are classified in [4] and are a Gödel-like universe and the near-horizon limit of the rotating BMPV black hole [61]. ${ }^{13}$ The latter family of solutions contains the $A d S_{2} \times S^{3}$ and $A d S_{3} \times S^{2}$ solutions as special cases. Even though there are maximally supersymmetric solutions which are not locally symmetric, they all happen to be homogeneous space-times $[9,56,63]$. It is also interesting to note that the maximally supersymmetric solutions of the theories with 8 real supercharges in $D=4,5,6$ dimensions are related via dimensional reduction or oxidation [9, 64].

\footnotetext{
${ }^{11}$ They have to be locally isometric to a product of a Riemannian symmetric space times a Minkowskian, $\mathrm{dS}$, AdS or Hpp-wave geometry.

${ }^{12}$ Notice that in $D=4$ dimensions $F^{\hat{\alpha}_{2}}$ itself is not necessarily decomposable. Instead we have to split it into a complex self-dual and anti-self-dual part and use the appropriate form of the second decomposition in (4.11).

${ }^{13}$ In [4] three additional solutions have been found but were left unidentified, it was shown in [62] that they also belong to the family of near-horizon BMPV solutions. See also [9].
} 


\begin{tabular}{|c|c|c|c|c|c|c|}
\hline dim. & SUSY & $q$ & \multicolumn{2}{|c|}{$\mathrm{AdS} \times S$} & $\mathrm{H} p p$-wave & others \\
\hline$D=11$ & $\mathcal{N}=1$ & 32 & $\begin{array}{l}\mathrm{AdS}_{4} \times S^{7} \\
\mathrm{AdS}_{7} \times S^{4}\end{array}$ & [40] & $\mathrm{KG}_{11}[41]$ & - \\
\hline$D=10$ & IIB & 32 & $\mathrm{AdS}_{5} \times S^{5}$ & {$[52,53]$} & $\mathrm{KG}_{10}[65]$ & - \\
\hline$D=6$ & $\begin{array}{l}\mathcal{N}=(2,0) \\
\mathcal{N}=(1,0)\end{array}$ & $\begin{array}{c}16 \\
8\end{array}$ & $\mathrm{AdS}_{3} \times S^{3}$ & {$[66]$} & $\mathrm{KG}_{6}[67]$ & - \\
\hline$D=5$ & $\mathcal{N}=2$ & 8 & $\begin{array}{l}\mathrm{AdS}_{2} \times S^{3} \\
\mathrm{AdS}_{3} \times S^{2}\end{array}$ & {$[66,68]$} & $\mathrm{KG}_{5}[67]$ & $\begin{array}{c}\text { Gödel-like [4], } \\
\text { NH-BMPV }[69,70]\end{array}$ \\
\hline$D=4$ & $\mathcal{N}=2$ & 8 & $\mathrm{AdS}_{2} \times S^{2}$ & {$[71,72]$} & $\mathrm{KG}_{4}[42]$ & - \\
\hline
\end{tabular}

Table 2. All possible maximally supersymmetric solutions with non-trivial flux; $q$ denotes the number of real supercharges, cf. [73].

\section{Conclusions}

In this paper we studied maximally supersymmetric solutions of all supergravities in spacetime dimensions $3 \leq D \leq 11$ - including gauged supergravities as well as supergravities with background fluxes. We found that the maximally supersymmetric solutions generically split into three separate classes. First of all there are the ungauged and undeformed supergravities without fluxes and a $D$-dimensional Minkowskian background. The second class of solutions consists of backgrounds without fluxes but the supergravity is gauged or otherwise deformed. In this case the Killing spinor equations are straightforward to integrate, implying that the space-time is maximally symmetric and therefore either again Minkowskian or $\operatorname{AdS}_{D}$. There are however certain algebraic conditions (3.8) which the fermionic shift-matrices $A_{0}, A_{1}$ and $A_{2}$ have to satisfy and which restrict the possible gaugings or deformations.

The third class of solutions has non-trivial background fluxes. This requires that all shift matrices $A_{0}, A_{1}, A_{2}$ vanish and the R-symmetry connection has no background value. It implies that the fermionic supersymmetry variations take exactly the same form as for the corresponding ungauged theories. Moreover, this class of solutions can only exist if either the gravitational multiplet has no spin- $\frac{1}{2}$ fermions or the theory is chiral. This selects among all supergravities the ones listed in table 1 and in addition selects the possible fluxes. Using the correspondence with the ungauged theories we argued that for all these theories all solutions are known and classified; we list them in table 2. One aspect of our analysis was to show that this list is exhaustive.

Of course in certain cases solutions from different classes can be related to each other. The solutions of the form $\mathrm{AdS}_{d} \times S^{(D-d)}$ — which might arise in ungauged theories — can be truncated to an effective $d$-dimensional description in terms of a gauged supergravity with an $\mathrm{AdS}_{d}$ background. The gauge group in this case is $\mathrm{SO}(D-d+1)$, i.e. the isometry group of the sphere $S^{(D-d)}$. At the same time not every AdS-solution of a gauged supergravity can be obtained from a sphere compactification. 
As a technical by-product of our analysis we derived the general formula (C.8) for the gauging of the R-symmetry in maximally supersymmetric solutions in dimensions $D \geq 4$. It shows that in the background the R-symmetry can only be gauged by vector fields from the gravitational multiplet (i.e. graviphotons) and that this gauging is completely fixed by the first shift matrix $A_{0}$ and therefore uniquely determined.

\section{Acknowledgments}

This work was supported by the German Science Foundation (DFG) under the Collaborative Research Center (SFB) 676 "Particles, Strings and the Early Universe" and the Research Training Group (RTG) 1670 "Mathematics inspired by String Theory and Quantum Field Theory".

We have benefited from conversations and correspondence with Eric Bergshoeff, Constantin Muranaka, Hagen Triendl and Dan Waldram.

\section{A $\quad \Gamma$-matrix conventions}

In this appendix we collect some useful $\Gamma$-matrix identities used throughout the paper. We mainly follow the definitions and conventions of [74]. The $\Gamma^{M}$ are defined via their anti-commutation relation

$$
\Gamma^{M} \Gamma^{N}+\Gamma^{N} \Gamma^{M}=2 g^{M N} \mathbb{1} .
$$

Frequently in the main text their antisymmetric products appear and we abbreviate

$$
\Gamma^{M_{1} \ldots M_{p}}=\Gamma^{\left[M_{1}\right.} \ldots \Gamma^{\left.M_{p}\right]},
$$

where the antisymmetrization [...] is with total weight 1 , i.e. $\Gamma^{M N}=\frac{1}{2}\left(\Gamma^{M} \Gamma^{N}-\Gamma^{N} \Gamma^{M}\right)$. In even dimensions $D=2 m$ we additionally have the chirality operator $\Gamma_{*}$ defined by

$$
\Gamma_{*}=(-i)^{m+1} \Gamma_{0} \Gamma_{1} \ldots \Gamma_{D-1}
$$

From its definition one infers [74]

$$
\Gamma^{M_{1} \ldots M_{p}} \Gamma_{*}=-(-i)^{m+1} \frac{1}{(D-p) !} \epsilon^{M_{p} \ldots M_{1}} N_{1} \ldots N_{D-p} \Gamma^{N_{1} \ldots N_{D-p}}
$$

while in odd dimensions $D=2 m+1$ one has instead

$$
\Gamma^{M_{1} \ldots M_{p}}=i^{m+1} \frac{1}{(D-p) !} \epsilon^{M_{1} \ldots M_{p}} N_{D-p} \ldots N_{1} \Gamma^{N_{1} \ldots N_{D-p}} .
$$

In even dimensions all anti-symmetric products $\Gamma^{M_{1} \ldots M_{p}}$ are linearly independent whereas in odd dimensions this only holds for $p \leq m$ due to (A.5). Moreover we denote the contraction with $\Gamma$-matrices by a dot ".", i.e. for a $p$-form $F$ we define

$$
F \cdot \Gamma=F_{M_{1} \ldots M_{p}} \Gamma^{M_{1} \ldots M_{p}} .
$$




\section{B Analysis of the integrability condition}

In this appendix we analyze the integrability condition (4.4) and argue that for all the theories listed in table 1 the term $\mathcal{H}_{M N}$ can be the only term at zeroth order in the $\Gamma$-matrices and has therefore to vanish in a maximally supersymmetric background.

Let us first note that all the theories in table 1 only allow for background fluxes $F^{\hat{\alpha}_{p}}$ for one particular value of $p$, so the expression (2.7) for $\mathcal{F}_{M}$ simplifies as we do not have to sum over different values for $p$. We want to inspect (4.4) term by term. While the Riemann tensor $R_{M N P Q}$ enters only at the quadratic order in $\Gamma$, also the third term $\left(\mathrm{D}_{[M} \mathcal{F}_{N]}+\mathrm{D}_{[M} A_{0} \Gamma_{N]}\right)$ cannot contain any terms at zeroth order in $\Gamma$ as can be directly seen from (2.7) and (2.8) with $p>1$. To analyze the remaining term in (4.4) we notice that this term can only produce something of vanishing order in $\Gamma$ from the anti-commutator of two equal powers of $\Gamma$-matrices, i.e.

$$
\left\{\Gamma^{M_{1} \ldots M_{r}}, \Gamma_{N_{1} \ldots N_{r}}\right\}=p ! \delta_{N_{r}}^{\left[M_{1}\right.} \ldots \delta_{N_{1}}^{\left.M_{r}\right]}+\ldots
$$

where the dots denote terms of higher order in $\Gamma$. On the hand the corresponding commutator yields at least a term quadratic in $\Gamma$ and also the (anti-)commutator of two different powers of $\Gamma$-matrices cannot give anything at zeroth order. With this knowledge we can finally compute the last term in (4.4) to find

$$
\begin{aligned}
&\left(\left(\mathcal{F}_{M}+A_{0} \Gamma_{M}\right)(\right.\left.\left.\mathcal{F}_{N}+A_{0} \Gamma_{N}\right)-(M \leftrightarrow N)\right)= \\
&=\left[\mathcal{F}_{M}, \mathcal{F}_{N}\right]+\left[\mathcal{F}_{M}, A_{0} \Gamma_{N}\right]-A_{0}\left[\mathcal{F}_{N}, A_{0} \Gamma_{M}\right]+2 A_{0} A_{0} \Gamma_{M N} \\
&=\frac{1}{2}(p-1) !\left(\beta_{(p)}^{2}-p^{2}\right)\left[B_{\hat{\alpha}_{p}}, B_{\hat{\beta}_{p}}\right] F_{M P_{1} \ldots P_{p-1}}^{\hat{\alpha}_{p}} F_{N}^{\hat{\beta}_{p}} P_{p-1} \ldots P_{p} \\
& \quad \quad+\delta_{p, 2} 4(D-3)\left[B_{\hat{\alpha}_{2}}, A_{0}\right] F_{M N}^{\hat{\alpha}_{2}}+\ldots,
\end{aligned}
$$

where we suppressed the indices $(i, j, \ldots)$ and the dots denote again higher order terms. For the computation of the commutator $\left[\mathcal{F}_{M}, \mathcal{F}_{N}\right]$ we used $(2.7),($ B.1) and

$$
\begin{aligned}
& {\left[B_{\hat{\alpha}_{p}} \Gamma^{M_{1} \ldots M_{r}}, B_{\hat{\beta}_{p}} \Gamma^{N_{1} \ldots N_{r}}\right]} \\
& \quad=\frac{1}{2}\left(\left[B_{\hat{\alpha}_{p}}, B_{\hat{\beta}_{p}}\right]\left\{\Gamma^{M_{1} \ldots M_{r}}, \Gamma^{N_{1} \ldots N_{r}}\right\}+\left\{B_{\hat{\alpha}_{p}}, B_{\hat{\beta}_{p}}\right\}\left[\Gamma^{M_{1} \ldots M_{r}}, \Gamma^{N_{1} \ldots N_{r}}\right]\right) .
\end{aligned}
$$

For all the theories where $\hat{\alpha}_{p}$ can take only one possible value the commutator $\left[B_{\hat{\alpha}_{p}}, B_{\hat{\beta}_{p}}\right]$ on the right hand side of (B.2) clearly vanishes. Moreover in this case $B_{\hat{\alpha}_{p}}$ is proportional to the unit matrix, therefore also the second commutator $\left[B_{\hat{\alpha}_{2}}, A_{0}\right]$ vanishes. The only theory in table 1 for which $\hat{\alpha}_{p}$ can take multiple values is the six-dimensional $\mathcal{N}=(2,0)$ theory. But here $p=D / 2=3$ and hence using (2.9) we have $\beta_{(p)}=p$ so that also in this case the terms at zeroth order in $\Gamma$ vanish.

It remains to check that in odd dimensions $D$ there are also no terms of order $D$ in $\Gamma$. These could be dualized into zero order terms using (A.5). Since we can restrict the analysis to $p<\frac{D}{2}$ it is clear that such terms cannot arise from $\mathrm{D}_{[M} \mathcal{F}_{N]}$ or $\left[\mathcal{F}_{M} A_{0}, \Gamma_{N}\right]$ as can be seen from the definition (2.7). The commutator $\left[\mathcal{F}_{M}, \mathcal{F}_{N}\right]$ can however produce only terms of even order in $\Gamma$. 


\section{The gauged R-symmetry connection}

In this appendix we show that in a maximally supersymmetric background the R-symmetry can only be gauged by vector fields from the gravity multiplet (graviphotons). ${ }^{14}$ To be more specific, we will show that a non-vanishing background value for those moment maps $t_{\tilde{\alpha}_{2}}$ from (2.14) which couple to the vector fields from other multiplets (i.e. vector multiplets) is not compatible with unbroken supersymmetry. We should stress that this is only a statement about the maximally supersymmetric background, at an arbitrary point in field space these restrictions on the gaugings do not necessarily need to be satisfied. In other words, a theory in which there are gauge fields that do not belong to the gravity multiplet might still admit maximally supersymmetric vacua. Note that the following analysis does not rely on any specific formulation of a gauged supergravity and is valid in any dimension $D>3$.

As explained in section 2 the R-symmetry connection $Q_{M}$ generically splits into a pure scalar dependent part $Q_{M}^{\text {scalar }}$ and a gauge field depend part $Q_{M}^{\text {gauge }}$. The latter can be expressed in terms of the gauge fields $A^{\alpha_{2}}$ as

$$
\left(Q_{M}^{\text {gauge }}\right)_{j}^{i}=A_{M}^{\alpha_{2}}\left(t_{\alpha_{2}}\right)_{j}^{i}
$$

Equivalently the corresponding part of the field strength $\mathcal{H}_{M N}$ reads $\mathcal{H}_{M N}^{\text {gauge }}=F_{M N}^{\alpha_{2}} t_{\alpha_{2}}$. In the following we determine the explicit form of the matrices $t_{\alpha_{2}}$ in the maximally supersymmetric background, i.e. for vanishing fermionic shift matrices $A_{1}$ and $A_{2}$.

The field strength $\mathcal{H}_{M N}$ enters the supersymmetry variation of the Lagrangian via the kinetic term for the gravitini which always takes the form

$$
e^{-1} \mathcal{L}_{\bar{\psi} \partial \psi}=-\frac{1}{2} \bar{\psi}_{i M} \Gamma^{M N P} \mathrm{D}_{N} \psi_{P}^{i}
$$

Inserting into this the supersymmetry variation (2.5) of $\psi_{M}^{i}$ produces a term of the form

$$
e^{-1} \delta \mathcal{L}_{\bar{\psi} \partial \psi}=\frac{1}{2}\left(\mathcal{H}_{M N}^{\text {gauge }}\right)_{j}^{i} \bar{\psi}_{i P} \Gamma^{M N P} \epsilon^{j}+\ldots
$$

To read off $\mathcal{H}_{M N}$ we collect all possible terms which produce similar terms under a supersymmetry transformation. If we demand $A_{1}=A_{2}=0$ these are given by

$$
\begin{aligned}
e^{-1} \mathcal{L}_{\bar{\psi} \psi} & =\frac{1}{2} d_{0} A_{0 j}^{i} \bar{\psi}_{i M} \Gamma^{M N} \psi_{N}^{j}, \\
e^{-1} \mathcal{L}_{F \bar{\psi} \psi} & =\frac{1}{2} e_{0} F_{M N}^{\hat{\alpha}_{2}}\left(B_{\hat{\alpha}_{2}}\right)_{j}^{i} \bar{\psi}_{i}^{P} \Gamma_{[P} \Gamma^{M N} \Gamma_{R]} \psi^{j R},
\end{aligned}
$$

where the numerical constants $d_{0}$ and $e_{0}$ are fixed by supersymmetry and can be determined to take the values

$$
d_{0}=-e_{0}=(D-2) .
$$

From (2.5) it follows that the supersymmetry variations of (C.4) contain precisely terms of the required form

$$
e^{-1} \delta \mathcal{L}_{\bar{\psi} \psi}=d_{0} F_{M N}^{\hat{\alpha}_{2}} A_{0 j}^{i}\left(B_{\hat{\alpha}_{2}}\right)_{k}^{j} \bar{\psi}_{i}^{P}\left(-(D-3) \Gamma^{M N}{ }_{P}+2 \delta_{P}^{[M} \Gamma^{N]}\right) \epsilon^{k}+\ldots,
$$

\footnotetext{
${ }^{14}$ See also the discussion below equation $(2.14)$.
} 
and

$$
e^{-1} \delta \mathcal{L}_{F \bar{\psi} \psi}=e_{0} F_{M N}^{\hat{\alpha}_{2}}\left(B_{\hat{\alpha}_{2}}\right)_{j}^{i} A_{0 k}^{j} \bar{\psi}_{i}^{P}\left((D-3) \Gamma^{M N}{ }_{P}+2 \delta_{P}^{[M} \Gamma^{N]}\right) \epsilon^{k}+\ldots
$$

The terms cubic in $\Gamma$-matrices have to cancel (C.3), so we finally determine

$$
t_{\hat{\alpha}_{2}}=2(D-2)(D-3)\left\{A_{0}, B_{\hat{\alpha}_{2}}\right\}, \quad t_{\tilde{\alpha}_{2}}=0 .
$$

This means in particular that in a maximally supersymmetric background the R-symmetry can only be gauged by the graviphotons $A^{\hat{\alpha}_{2}}$, but not by gauge fields $A^{\tilde{\alpha}_{2}}$ in additional vector multiplets.

Finally we want to argue that the matrices $t_{\hat{\alpha}_{2}}$ satisfying (C.8) span a Lie-subalgebra of the R-symmetry algebra $\mathfrak{g}_{R}$, i.e. that they close with respect to the Lie-bracket. Using the fact that we always need $A_{0}^{2} \sim \mathbb{1}$ in a maximally supersymmetric background (according to (3.8)), we immediately find that

$$
\left[t_{\hat{\alpha}_{2}}, A_{0}\right]=0,
$$

i.e. that $A_{0}$ is invariant under the adjoint action of the $t_{\hat{\alpha}_{2}}$. To proceed, let us denote the generators of $\mathfrak{g}_{R}$ by $T_{A}, A=1, \ldots, \operatorname{dim}\left(\mathfrak{g}_{R}\right)$. Now invariance of the gravitino variations (2.5) under R-symmetry transformations requires all the $B$-matrices to be $\mathfrak{g}_{R}$ invariant, in the sense that

$$
\left(T_{A}\right)_{\hat{\alpha}_{2}}{ }^{\hat{\beta}_{2}} B_{\hat{\beta}_{2}}-\left[T_{A}, B_{\hat{\alpha}_{2}}\right]=0 .
$$

Here $\left(T_{A}\right)_{\hat{\alpha}_{2}}{ }^{\hat{\beta}_{2}}$ and $\left(T_{A}\right)_{i}{ }^{j}$ denote the R-symmetry generators in the representations of the graviphotons and the gravitini respectively. So loosely speaking the $B$-matrices "translate" between different representations of $\mathfrak{g}_{R}$. As $t_{\alpha_{2}} \in \mathfrak{g}_{R}$ we can always find (generically scalar dependent) matrices $\Theta_{\alpha_{2}}{ }^{A}$ such that

$$
t_{\alpha_{2}}=\Theta_{\alpha_{2}}{ }^{A} T_{A}
$$

Using this information it follows from (C.9) and (C.10) that

$$
\begin{aligned}
{\left[t_{\hat{\alpha}_{2}}, t_{\hat{\beta}_{2}}\right] } & =2(D-2)(D-3)\left\{A_{0},\left[t_{\hat{\alpha}_{2}}, B_{\hat{\beta}_{2}}\right]\right\} \\
& =2(D-2)(D-3)\left\{A_{0},\left(t_{\hat{\alpha}_{2}}\right)_{\hat{\beta}_{2}} \hat{\gamma}_{2} B_{\hat{\gamma}_{2}}\right\} \\
& =\left(t_{\hat{\alpha}_{2}}\right)_{\hat{\beta}_{2}}{ }^{\hat{\gamma}_{2}} t_{\hat{\gamma}_{2}},
\end{aligned}
$$

where we have introduced $\left(t_{\hat{\alpha}_{2}}\right)_{\hat{\beta}_{2}}{ }^{\hat{\gamma}_{2}}=\Theta_{\hat{\alpha}_{2}}{ }^{A}\left(T_{A}\right)_{\hat{\beta}_{2}}{ }^{\hat{\gamma}_{2}}$.

Open Access. This article is distributed under the terms of the Creative Commons Attribution License (CC-BY 4.0), which permits any use, distribution and reproduction in any medium, provided the original author(s) and source are credited.

\section{References}

[1] K.P. Tod, All Metrics Admitting Supercovariantly Constant Spinors, Phys. Lett. B 121 (1983) 241 [INSPIRE].

[2] K. Behrndt, D. Lüst and W.A. Sabra, Stationary solutions of $N=2$ supergravity, Nucl. Phys. B 510 (1998) 264 [hep-th/9705169] [INSPIRE]. 
[3] J.P. Gauntlett, D. Martelli, S. Pakis and D. Waldram, G structures and wrapped NS5-branes, Commun. Math. Phys. 247 (2004) 421 [hep-th/0205050] [INSPIRE].

[4] J.P. Gauntlett, J.B. Gutowski, C.M. Hull, S. Pakis and H.S. Reall, All supersymmetric solutions of minimal supergravity in five- dimensions, Class. Quant. Grav. 20 (2003) 4587 [hep-th/0209114] [INSPIRE].

[5] J.M. Figueroa-O'Farrill and G. Papadopoulos, Maximally supersymmetric solutions of ten-dimensional and eleven-dimensional supergravities, JHEP 03 (2003) 048 [hep-th/0211089] [INSPIRE].

[6] J.P. Gauntlett and S. Pakis, The geometry of D = 11 Killing spinors, JHEP 04 (2003) 039 [hep-th/0212008] [INSPIRE].

[7] J.P. Gauntlett, D. Martelli and D. Waldram, Superstrings with intrinsic torsion, Phys. Rev. D 69 (2004) 086002 [hep-th/0302158] [INSPIRE].

[8] J.B. Gutowski, D. Martelli and H.S. Reall, All Supersymmetric solutions of minimal supergravity in six-dimensions, Class. Quant. Grav. 20 (2003) 5049 [hep-th/0306235] [INSPIRE].

[9] A. Chamseddine, J.M. Figueroa-O'Farrill and W. Sabra, Supergravity vacua and Lorentzian Lie groups, hep-th/0306278 [INSPIRE].

[10] J.P. Gauntlett, J.B. Gutowski and S. Pakis, The geometry of D $=11$ null Killing spinors, JHEP 12 (2003) 049 [hep-th/0311112] [INSPIRE].

[11] J.P. Gauntlett, D. Martelli, J. Sparks and D. Waldram, Supersymmetric AdS $S_{5}$ solutions of M-theory, Class. Quant. Grav. 21 (2004) 4335 [hep-th/0402153] [InSPIRE].

[12] G. Dall'Agata, On supersymmetric solutions of type IIB supergravity with general fluxes, Nucl. Phys. B 695 (2004) 243 [hep-th/0403220] [INSPIRE].

[13] M. Cariglia and O.A.P. Mac Conamhna, Timelike Killing spinors in seven dimensions, Phys. Rev. D 70 (2004) 125009 [hep-th/0407127] [INSPIRE].

[14] J.P. Gauntlett and J.B. Gutowski, General concentric black rings, Phys. Rev. D 71 (2005) 045002 [hep-th/0408122] [INSPIRE].

[15] J. Gillard, U. Gran and G. Papadopoulos, The spinorial geometry of supersymmetric backgrounds, Class. Quant. Grav. 22 (2005) 1033 [hep-th/0410155] [INSPIRE].

[16] J.P. Gauntlett, D. Martelli, J. Sparks and D. Waldram, Supersymmetric AdS backgrounds in string and M-theory, IRMA Lect. Math. Theor. Phys. 8 (2005) 217 [hep-th/0411194] [INSPIRE].

[17] U. Gran, J. Gutowski and G. Papadopoulos, The spinorial geometry of supersymmetric IIB backgrounds, Class. Quant. Grav. 22 (2005) 2453 [hep-th/0501177] [INSPIRE].

[18] J.B. Gutowski and W. Sabra, General supersymmetric solutions of five-dimensional supergravity, JHEP 10 (2005) 039 [hep-th/0505185] [INSPIRE].

[19] J. Bellorín and T. Ortín, All the supersymmetric configurations of $N=4, D=4$ supergravity, Nucl. Phys. B 726 (2005) 171 [hep-th/0506056] [INSPIRE].

[20] T. Ishino, H. Kodama and N. Ohta, Time-dependent solutions with null Killing spinor in M-theory and superstrings, Phys. Lett. B 631 (2005) 68 [hep-th/0509173] [INSPIRE].

[21] P. Meessen and T. Ortín, The supersymmetric configurations of $N=2, D=4$ supergravity coupled to vector supermultiplets, Nucl. Phys. B 749 (2006) 291 [hep-th/0603099] [INSPIRE]. 
[22] M. Huebscher, P. Meessen and T. Ortín, Supersymmetric solutions of $N=2 D=4$ SUGRA: The whole ungauged shebang, Nucl. Phys. B 759 (2006) 228 [hep-th/0606281] [INSPIRE].

[23] J. Bellorín, P. Meessen and T. Ortín, All the supersymmetric solutions of $N=1, d=5$ ungauged supergravity, JHEP 01 (2007) 020 [hep-th/0610196] [INSPIRE].

[24] S.L. Cacciatori, M.M. Caldarelli, D. Klemm, D.S. Mansi and D. Roest, Geometry of four-dimensional Killing spinors, JHEP 07 (2007) 046 [arXiv:0704.0247] [INSPIRE].

[25] P. Meessen, T. Ortín and S. Vaula, All the timelike supersymmetric solutions of all ungauged $D=4$ supergravities, JHEP 11 (2010) 072 [arXiv:1006.0239] [INSPIRE].

[26] M. Akyol and G. Papadopoulos, Spinorial geometry and Killing spinor equations of $6-D$ supergravity, Class. Quant. Grav. 28 (2011) 105001 [arXiv: 1010.2632] [INSPIRE].

[27] J.P. Gauntlett and J.B. Gutowski, All supersymmetric solutions of minimal gauged supergravity in five-dimensions, Phys. Rev. D 68 (2003) 105009 [Erratum ibid. D 70 (2004) 089901] [hep-th/0304064] [INSPIRE].

[28] M.M. Caldarelli and D. Klemm, All supersymmetric solutions of $N=2, D=4$ gauged supergravity, JHEP 09 (2003) 019 [hep-th/0307022] [INSPIRE].

[29] J.B. Gutowski and H.S. Reall, General supersymmetric AdS $S_{5}$ black holes, JHEP 04 (2004) 048 [hep-th/0401129] [INSPIRE].

[30] M. Cariglia and O.A.P. Mac Conamhna, The general form of supersymmetric solutions of $N=(1,0) \mathrm{U}(1)$ and $\mathrm{SU}(2)$ gauged supergravities in six-dimensions, Class. Quant. Grav. 21 (2004) 3171 [hep-th/0402055] [INSPIRE].

[31] S.L. Cacciatori, M.M. Caldarelli, D. Klemm and D.S. Mansi, More on BPS solutions of $N=2, D=4$ gauged supergravity, JHEP 07 (2004) 061 [hep-th/0406238] [INSPIRE].

[32] J. Bellorín and T. Ortín, Characterization of all the supersymmetric solutions of gauged $N=1, D=5$ supergravity, JHEP 08 (2007) 096 [arXiv:0705.2567] [INSPIRE].

[33] S.L. Cacciatori, D. Klemm, D.S. Mansi and E. Zorzan, All timelike supersymmetric solutions of $N=2, D=4$ gauged supergravity coupled to abelian vector multiplets, JHEP 05 (2008) 097 [arXiv: 0804.0009] [INSPIRE].

[34] M. Huebscher, P. Meessen, T. Ortín and S. Vaula, $N=2$ Einstein-Yang-Mills's BPS solutions, JHEP 09 (2008) 099 [arXiv: 0806.1477] [INSPIRE].

[35] J. Bellorín, Supersymmetric solutions of gauged five-dimensional supergravity with general matter couplings, Class. Quant. Grav. 26 (2009) 195012 [arXiv:0810.0527] [INSPIRE].

[36] D. Klemm and E. Zorzan, All null supersymmetric backgrounds of $N=2, D=4$ gauged supergravity coupled to abelian vector multiplets, Class. Quant. Grav. 26 (2009) 145018 [arXiv:0902.4186] [INSPIRE].

[37] K. Hristov, H. Looyestijn and S. Vandoren, Maximally supersymmetric solutions of $D=4$ $N=2$ gauged supergravity, JHEP 11 (2009) 115 [arXiv:0909.1743] [INSPIRE].

[38] D. Klemm and E. Zorzan, The timelike half-supersymmetric backgrounds of $N=2, D=4$ supergravity with Fayet-Iliopoulos gauging, Phys. Rev. D 82 (2010) 045012 [arXiv: 1003.2974] [INSPIRE].

[39] P. Meessen and T. Ortín, Supersymmetric solutions to gauged $N=2 D=4$ SUGRA: the full timelike shebang, Nucl. Phys. B 863 (2012) 65 [arXiv:1204.0493] [InSPIRE]. 
[40] P.G.O. Freund and M.A. Rubin, Dynamics of Dimensional Reduction, Phys. Lett. B 97 (1980) 233 [INSPIRE].

[41] J. Kowalski-Glikman, Vacuum States in Supersymmetric Kaluza-Klein Theory, Phys. Lett. B 134 (1984) 194 [INSPIRE].

[42] J. Kowalski-Glikman, Positive Energy Theorem And Vacuum States For The Einstein-Maxwell System, Phys. Lett. B 150 (1985) 125 [InSPIRE].

[43] H. Samtleben, Lectures on Gauged Supergravity and Flux Compactifications, Class. Quant. Grav. 25 (2008) 214002 [arXiv: 0808.4076] [INSPIRE].

[44] I.A. Bandos and T. Ortín, On the dualization of scalars into (d-2)-forms in supergravity. Momentum maps, R-symmetry and gauged supergravity, JHEP 08 (2016) 135 [arXiv: 1605.05559] [INSPIRE].

[45] M. Trigiante, Gauged Supergravities, arXiv:1609.09745 [INSPIRE].

[46] S. de Alwis, J. Louis, L. McAllister, H. Triendl and A. Westphal, Moduli spaces in AdS 4 supergravity, JHEP 05 (2014) 102 [arXiv:1312.5659] [INSPIRE].

[47] J. Louis and H. Triendl, Maximally supersymmetric $A d S_{4}$ vacua in $N=4$ supergravity, JHEP 10 (2014) 007 [arXiv: 1406.3363] [INSPIRE].

[48] J. Louis and S. Lüst, Supersymmetric $A d S_{7}$ backgrounds in half-maximal supergravity and marginal operators of $(1,0)$ SCFTs, JHEP 10 (2015) 120 [arXiv:1506.08040] [INSPIRE].

[49] J. Louis, $\mathrm{H}$. Triendl and M. Zagermann, $\mathcal{N}=4$ supersymmetric $A d S_{5}$ vacua and their moduli spaces, JHEP 10 (2015) 083 [arXiv:1507.01623] [INSPIRE].

[50] J. Louis and C. Muranaka, Moduli spaces of $A d S_{5}$ vacua in $\mathcal{N}=2$ supergravity, JHEP 04 (2016) 178 [arXiv : 1601.00482] [INSPIRE].

[51] W. Nahm, Supersymmetries and their Representations, Nucl. Phys. B 135 (1978) 149 [INSPIRE].

[52] J.H. Schwarz, Covariant Field Equations of Chiral $N=2 D=10$ Supergravity, Nucl. Phys. B 226 (1983) 269 [INSPIRE].

[53] J.H. Schwarz and P.C. West, Symmetries and Transformations of Chiral $N=2 D=10$ Supergravity, Phys. Lett. B 126 (1983) 301 [INSPIRE].

[54] H. Nishino and E. Sezgin, Matter and Gauge Couplings of $N=2$ Supergravity in Six-Dimensions, Phys. Lett. B 144 (1984) 187 [INSPIRE].

[55] M. Awada, P.K. Townsend and G. Sierra, Six-dimensional Simple and Extended Chiral Supergravity in Superspace, Class. Quant. Grav. 2 (1985) L85 [INSPIRE].

[56] M. Cahen and N. Wallach, Lorentzian symmetric spaces, Bull. Am. Math. Soc. 76 (1970) 585.

[57] R. Penrose, Any space-time has a plane wave as a limit, in Differential geometry and relativity, Reidel, Dordrecht The Netherlands (1976), pp. 271-275.

[58] R. Güven, Plane wave limits and T duality, Phys. Lett. B 482 (2000) 255 [hep-th/0005061] [INSPIRE].

[59] M. Blau, J.M. Figueroa-O'Farrill, C. Hull and G. Papadopoulos, Penrose limits and maximal supersymmetry, Class. Quant. Grav. 19 (2002) L87 [hep-th/0201081] [INSPIRE].

[60] M. Blau, J.M. Figueroa-O'Farrill and G. Papadopoulos, Penrose limits, supergravity and brane dynamics, Class. Quant. Grav. 19 (2002) 4753 [hep-th/0202111] [INSPIRE]. 
[61] J.C. Breckenridge, R.C. Myers, A.W. Peet and C. Vafa, D-branes and spinning black holes, Phys. Lett. B 391 (1997) 93 [hep-th/9602065] [INSPIRE].

[62] B. Fiol, C. Hofman and E. Lozano-Tellechea, Causal structure of D $=5$ vacua and axisymmetric space-times, JHEP 02 (2004) 034 [hep-th/0312209] [INSPIRE].

[63] N. Alonso-Alberca, E. Lozano-Tellechea and T. Ortín, The near-horizon limit of the extreme rotating D $=5$ black hole as a homogeneous space-time, Class. Quant. Grav. 20 (2003) 423 [hep-th/0209069] [INSPIRE].

[64] E. Lozano-Tellechea, P. Meessen and T. Ortín, On $D=4, D=5, D=6$ vacua with eight supercharges, Class. Quant. Grav. 19 (2002) 5921 [hep-th/0206200] [INSPIRE].

[65] M. Blau, J.M. Figueroa-O'Farrill, C. Hull and G. Papadopoulos, A new maximally supersymmetric background of IIB superstring theory, JHEP 01 (2002) 047 [hep-th/0110242] [INSPIRE].

[66] G.W. Gibbons, G.T. Horowitz and P.K. Townsend, Higher dimensional resolution of dilatonic black hole singularities, Class. Quant. Grav. 12 (1995) 297 [hep-th/9410073] [INSPIRE].

[67] P. Meessen, A small note on P P wave vacua in six-dimensions and five-dimensions, Phys. Rev. D 65 (2002) 087501 [hep-th/0111031] [INSPIRE].

[68] A.H. Chamseddine, S. Ferrara, G.W. Gibbons and R. Kallosh, Enhancement of supersymmetry near 5-D black hole horizon, Phys. Rev. D 55 (1997) 3647 [hep-th/9610155] [INSPIRE].

[69] M. Cvetič and F. Larsen, Near horizon geometry of rotating black holes in five-dimensions, Nucl. Phys. B 531 (1998) 239 [hep-th/9805097] [INSPIRE].

[70] J.P. Gauntlett, R.C. Myers and P.K. Townsend, Black holes of D $=5$ supergravity, Class. Quant. Grav. 16 (1999) 1 [hep-th/9810204] [INSPIRE].

[71] B. Bertotti, Uniform electromagnetic field in the theory of general relativity, Phys. Rev. 116 (1959) 1331 [INSPIRE].

[72] I. Robinson, A Solution of the Maxwell-Einstein Equations, Bull. Acad. Pol. Sci. Ser. Sci. Math. Astron. Phys. 7 (1959) 351 [inSPIRE].

[73] N. Alonso-Alberca and T. Ortín, Supergravity vacua today, gr-qc/0210039 [INSPIRE].

[74] D.Z. Freedman and A. Van Proeyen, Supergravity, Cambridge University Press, (2012). 